\title{
Enhancing Cognitive Resilience in Adolescence and Young Adults: A Neuroscience- informed Approach
}

\author{
Authors: Tara Rezapour ${ }^{1,2}$, Shervin Assari ${ }^{3}$, Namik Kirlic ${ }^{5}$, Jasmin Vassileva ${ }^{4}$, Hamed Ekhtiari ${ }^{5}$ \\ 1. Institute for Cognitive Science Studies, Tehran, Iran \\ 2. Iranian National Center for Addiction Studies, Tehran University of Medical Sciences, Tehran, Iran \\ 3. University of Medicine and Science, Los Angeles, CA, USA \\ 4. Department of Psychiatry, Institute for Drug and Alcohol Studies, Virginia Commonwealth University, \\ Richmond, VA, USA \\ 5. Laureate Institute for Brain Research, Tulsa, OK, USA \\ Correspondence: Hamed Ekhtiari, MD,PhD, hekhtiari@laureateinstitute.org
}

\begin{abstract}
:
Resilience, as a trait, process, or outcome, is an important factor to explain behavioral diversity between individuals and population groups in face of stress and adversity. Individuals and groups who can bounce back shorty after stressful events, experience less severe negative emotions (depression, anxiety) and manage situations through efficient problem-solving strategies are categorized as resilient. Enhancing populations' and individuals' resilience becomes a central strategy for prevention of maladaptive behaviors, especially among adolescents. Several psychosocial interventions, mostly taking a positive psychology approach, improve resilience and reduce disruptive behaviors (e.g., using illicit drug and alcohol or selfharm behaviors) among adolescents. However, the role of brain awareness and training interventions targeting cognitive underpinning of resilience is not fully explored. In this chapter, we firstly review the existing literature and address the interventions that indirectly increase cognitive resilience among schoolaged adolescents. Then we introduce the Promoting Cognitive Resilience (ProCoRe), a new multi-modal cognitive resilience training program, that taps different cognitive functions that are documented to be effective in the neuroscience literature. Clinical and public health implications of the ProCoRe as a prevention program to empower adolescents to avoid high risk behaviors in face of stressful through effective emotion regulation and impulse control. are discussed.
\end{abstract}

Key words: resilience, stress, substance use, cognitive resilience, ProCoRe, adolescence

\section{Introduction}

There has been a spiked interest in studying youth mental health problems, which affect nearly $20 \%$ of total adolescent population globally (World Health Organization, 2016). Moreover, it has been widely accepted that mental health disorders such as depression, suicide, eating disorders, and substance use disorders start to develop and manifest in adolescence and early adulthood (Chadda, 2018; Jurewicz, 2015; Nishida, Richards, \& Stafford, 2016). Bohm and Clayton (2019) documented a five-fold increase, from 2010 (831 cases) to 2016 ( 873 cases), in the prevalence of adolescent opioid-involved drug overdose deaths in the US. In 2018, the Substance Abuse and Mental Health Services Administration (SAMSHA) estimated 4.2 million adolescents (16.7\%) aged 12 to 17 were past-year illicit drug users, and about 1percent were current heavy drinkers. Importantly, these estimations increase significantly in young adults who are aged 18-25 years (prevalence of $38.7 \%$ and $9.0 \%$ for illicit drug and alcohol, respectively). Risk taking, using to enhance a positive state (positive reinforcement), using to cope with a negative affect or stress (negative reinforcement). Lack of self-control against peer pressure are frequently reported as the main reasons that adolescents start to use alcohol and other drugs (Donovan, 2004; Dow \& Kelly, 2013; Titus, Godley, \& White, 2007). These reasons raise an urgent need to develop proper mental health programs that can help protect adolescents from engagement in maladaptive behaviors in response to stressful, volatile and risky situations. 
Neurobiological evidence support a dual system model to describe the inclination of adolescents towards risk taking and emotion dis-regulation (Hartley \& Somerville, 2015). According to this model, adolescence is a critical life-course period associated with considerable developmental brain changes (Knudsen, 2004; Spear, 2000; Wetherill \& Tapert, 2013) characterized by continued structural and functional development of frontostriatal circuitry implicated in reward processing, impulse control, and emotion regulation(Bjork, Smith, Danube, \& Hommer, 2007; Galvan, Hare, Voss, Glover, \& Casey, 2007; Galvan et al., 2006). Frontostriatal circuitry is characterized by asynchronous pattern of development, with an impulsive socioemotional striatal system maturing early and being disproportionately active relative to later-maturing top-down cognitive control system mediated by the prefrontal cortex (Blakemore \& Robbins, 2012; B. J. Casey, Galvan, \& Hare, 2005; Clark \& Winters, 2002; Galvan et al., 2006; Giedd, 1999; Sowell et al., 2003). This asynchronous developmental pattern is related to increased risk-taking (L. Steinberg, 2004), preference for immediate gratification over long-term gains(Claus et al., 2018; Stanger, Budney, \& Bickel, 2013; L. Steinberg, Graham, S., O'Brien, L., Woolard, J., Cauffman, E., Banich, M., 2009), discounting of future negative consequences (L. Steinberg, Graham, S., O'Brien, L., Woolard, J., Cauffman, E., Banich, M., 2009), and exaggerated anticipation of positive outcomes of risky behaviors(Blakemore \& Robbins, 2012). All these aberrant cognitive processes place adolescents and young adults at much higher risk for impulsive, risk-taking, or maladaptive coping behaviors including problematic substance use and development of substance use disorders (SUDs). The influence of genetic factors also increases across adolescence, as individuals have more freedom to express their genetic predispositions for risky behaviors such as alcohol use (Dick, Adkins, \& Kuo, 2016; McGue M, 2006). Not surprisingly, adolescence is the peak age of onset for most of the psychiatric disorders (Lee et al., 2014). The transition from childhood to adolescence is accompanied by alterations in different levels of individual, interpersonal and social goals and relationships (Jaworska \& MacQueen, 2015). Moving from a small-scale society (primary school) to the complicated adult world exposes adolescents to a number of emotional challenges, evocative and risky situations (B. Casey, Jones, RM., Hare, TA., 2008). These stressful millstones, in turn, raise acute and chronic stress that may disturb physiological and psychological functions and increase the risk of development of serious pathological conditions (Dantzer, Cohen, Russo, \& Dinan, 2018; Franklin, Saab, \& Mansuy, 2012). Successful coping with these stressful conditions requires the ability to properly maintain mental homeostasis (e.g. emotion regulation and impulse control) as well as to actively employ effective problem solving and decision-making strategies (Dray, 2014).

In this context, the concept of "resilience" in adolescence has received increased attention among developmental, cognitive and clinical psychologists (Masten \& Barnes, 2018). Resilience includes various protective processes that enhance positive adaptation against stressful events (Liu, Zhang, Ji, \& Yang, 2018). These factors and processes conceptualized as enabling assets and resources map on to individual, family and culture (Masten \& Barnes, 2018). Therefore, resilience is defined as a dynamic capacity that buffers the impact of stress while keeping the balance in daily performance at both personal and societal levels (Wu et al., 2013). More recently, Masten and Barnes (2018) have extended this definition beyond individuals to include any complex adaptive system. According to their view, resilience could be applied to human organizations, ecosystems, economies, families, individuals as well as systems within an individual (e.g., immune system), however, in this chapter, we are focused on the resilience at the individual level.

Due to its multidimensional nature (Luthar SS, 2000; Wu et al., 2013), resilience can be discussed from different biological and psychological perspectives (Kahn, Collinge, \& Soltysik, 2016; Liu et al., 2018). A common thread among studies of the neurophysiological and neuropsychological mechanisms for resilience is its trainable nature. Various training programs focusing on positive psychology (Victoria Cerezo, OrtizTallo, Cardenal, \& De La Torre-Luque, 2014), flexible interpretation (Loprinzi CE, 2011), stress reduction (Goodman \& Schorling, 2012; Mealer et al., 2014), self-management (McCraty R, 2012), self-awareness (Dyrbye et al., 2016), self-care techniques and self-regulation (Blair \& Raver, 2015), emotion regulation, and decision making and social skills (Mache, Bernburg, Baresi, \& Groneberg, 2016), have been proposed to improve resilience (Joyce et al., 2018). Specific training programs within these psychological frameworks, show evidence for effectiveness to improve some aspects of resilience. Some examples are 
the Children's Resilience Program (Ambelu, Mulu, Seyoum, Ayalew, \& Hildrew, 2019), the Zippy's Friends (Clarke, Bunting, \& Barry, 2014), You Can Do It! Education (Yamamoto, Matsumoto, \& Bernard, 2017), the INSIGHTS (McClowry, Snow, Tamis-Lemonda, \& Rodriguez, 2010), and the Chicago School Readiness Project (CSRP)(Webster-Stratton, Jamila Reid, \& Stoolmiller, 2008).

In addition to the psychological approach to enhance resilience, a growing interest has emerged in the field of cognitive neuroscience towards studying resilience as a neurocognitive concept and defining its corresponding brain structures and functions (Richter, Kramer, Diekhof, \& Gruber, 2019). These studies have identified complex interactions between brain regions including the insula, cingulate cortex, hippocampus, nucleus accumbens, medial temporal lobe, and prefrontal cortex during emotion regulation and executive control (Iadipaolo et al., 2018; Liu et al., 2018; Russo, Murrough, Han, Charney, \& Nestler, 2012; Wu et al., 2013). The balanced interaction between various neural regions, networks, and processes underlying "cognitive resilience" is a novel target for exploration. Neuroscience-based cognitive resilience is defined as a set of brain-derived abilities and processes for coping with the negative consequences of stress, adversity and negative emotions while maintaining proper level of cognitive functions that are necessary for activities of daily living and avoiding high risk behaviors (Ram, Chandran, Sadar, \& Gowdappa, 2019). Cognitive resilience could be decomposed to various cognitive processes including working memory, response inhibition, cognitive flexibility, impulse control, reasoning, problem solving, planning and decision-making (Cambron et al., 2017; Nigg, 2017). So, for the successful control of thoughts, emotions, impulses and behaviors in the face of stressful events and evocative context, resilient adolescents should recruit a set of cognitive functions to encourage goal-attainment, positive adaptation, positive emotion, and adaptive decisions that will maximize long-term benefits (Artuch-Garde et al., 2017). From this perspective, a harmonized improvement in these cognitive processes will eventually result in higher resilience.

However, only a few studies have indirectly targeted cognitive resilience through uni- and multi-modal training of cognitive functions (e.g., attention, working memory, executive function) via curriculum-based interventions, exercise-based interventions, and social/interpersonal skills interventions among school-aged adolescents (Pandey A, 2018). Far transfer effect of training (transfer the effects of training to non-trained tasks)(Rossignoli-Palomeque, Perez-Hernandez, \& Gonzalez-Marques, 2018) include improvements in anxiety, teacher-reported behaviors, emotional symptoms (Roughan \& Hadwin, 2011), externalizing and internalizing behaviors (Riggs, 2006), impulsivity (Shure, 1993) and physical aggression(Grossman, 1997). In spite of the trainable components of cognitive resilience, we are not aware of any comprehensive cognitive resilience training programs for adolescents that target critical cognitive functions within a neuroscience-based framework, and trigger experience-expectant plasticity (EEP) (Fuhrmann, Knoll, \& Blakemore, 2015; Kerrr et al.,2011). EEP is a type of neuroplasticity that refers to processes whereby the brain expects certain stimuli to learn new skills or competencies. Adolescence is typically known as sensitive period due to high capacity for EEP (Fuhrmann, Knoll, \& Blakemore, 2015). A program in which training modules are developed based on real-life conditions, can trigger emotional or impulsive responses and train adaptive behaviors in a safe/controlled environment to develop EEP. In an ideal scenario, this program should provide adolescents with a neuroscience-based psychoeducation on cognitive components of resilience and instruct them on how to control their thoughts, emotions and behaviors by implementing metacognitive and compensatory strategies within the context of real-life scenarios. Games and cartoons should make the educational materials engaging and entertaining for adolescents.

To fill this knowledge gap, in the following sections, we will introduce a program that attempts to hierarchically train and integrate various dimensions of brain functions to build up cognitive resilience in adolescents. To our knowledge, this program is one of the first that takes a holistic approach to increase cognitive resilience of adolescents and young adults from a neurocognitive perspective.

\section{Promoting Cognitive Resilience (ProCoRe) for Resilience}

The "Promoting Cognitive Resilience" or shortly, ProCoRe is designed as a training program which targets cognitive functions involved in emotion-regulation, impulse-control, learning-skills, stress-management, planning, flexibility and problem-solving, known to be engaged in goal-directed adaptive behaviors(Kent, 
Rivers, \& Wrenn, 2015). ProCoRe is a paper-and-pencil program that integrates psychoeducational and game-based training. It includes 14 sessions. Each session takes around 90 minutes in a group setting. The curriculum content of each session is categorized into six interrelated sections including: brain challenge scenario (narrative), brain concept (psychoeducation), brain exercise (game), brain box (meta-cognitive training), brain tip (compensatory strategies) and brain planner (time-management and activity monitoring). This categorization is consistent across all sessions and presented below:

- Brain challenge scenarios: Each session starts with a cartoon-based scenario which narrates common problems that arise from deficits in the specific cognitive function targeted in the session (Debenham, Birrell, Champion, Askovic, \& Newton, 2020; Ekhtiari, Rezapour, Aupperle, \& Paulus, 2017). These scenarios explain how these cognitive deficits impact our everyday life. The most important feature of this part of the curriculum is using simple and first-person narrative sentences to increase their selfreference effect. For example, in session 9 that focuses on "emotional memory", we use a comic cartoon (Figure1) and narrated this scenario:

“.... I also experience strong and detailed memories related to previous stressful events. Travelling through these negative memories can lead to negative emotions, such as guilt, anxiety, or hopelessness. I wish that I could erase those memories or ignore the emotions that come along with them."

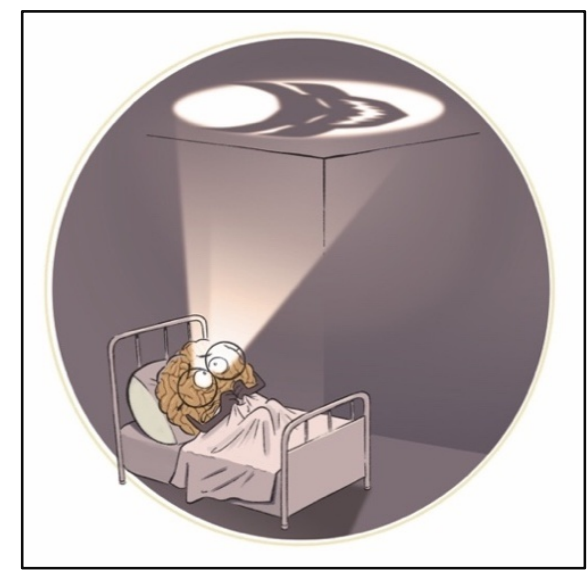

Figure 1. Sample of a cartoon used in the brain education part (session related to emotional memory) (Cartoons by NaeemTadayon, courtesy of Laureate Institute for Brain Research).

- Brain concepts: This section explains the cognitive function discussed in the session in a psychoeducational context and describes how the deficits/disorders involving this cognitive function affect our real life (Labriole, 2010). An example is emotional memory. It is explained that emotionally salient information particularly with negative valence, is more likely to be remembered for longer period of time rather than neutral information. Various interoceptive changes (e.g., heart rate) that may occur by recalling this information are also discussed. This section helps participants to gain awareness and insight about the underlying brain functions and the important roles they play in our day-to-day functioning.

- Brain tips: To efficiently manage cognitive resources, different evidence-based compensatory (i.e. stay focused strategies or mnemonics) or cognitive (i.e. eye gaze training or cognitive reappraisal) modulation strategies are provided as practical shortcuts that may help individuals to compensate for their limited cognitive and emotional resources especially in stressful conditions (Rossignoli-Palomeque, QuirosGodoy, Perez-Hernandez, \& Gonzalez-Marques, 2019; Vujovic \& Urry, 2018). For example, a strategy 
termed "expand your tunnel memory" is introduced to help participants expand their attentional span in face of distressing events and emotional memories. Using this brain tip may change the way that participants encode/recode information and reduce the intensity of the emotional response later when they recall the event or its memory. Such brain tips are usually followed by a relevant exercise (see next paragraph).

- Brain exercises: A set of game-based exercises is included in each session to engage relevant cognitive functions, as suggested by Kent et al., (2015). The additive architecture (see Figure2) is adapted to arrange the exercises across sessions, which means that each new session engages the previously activated cognitive functions plus the new one. The sessions have been ordered in such a way that they begin with the basic cognitive functions (e.g., attention and working memory) and gradually proceed to the higher order functions (e.g., episodic future thinking and prospective memory). Figure 3 shows a sample of a brain exercise that practices tunnel memory technique in session 9 that added affective memory to the episodic memory introduced in session 8 . This exercise is used to give participants an opportunity to experience how to reduce the vividness and emotionality of a central negative event in favor of the neutral details around. This exercise follows the preceding sections about the concept of tunnel memory and its modification strategies.

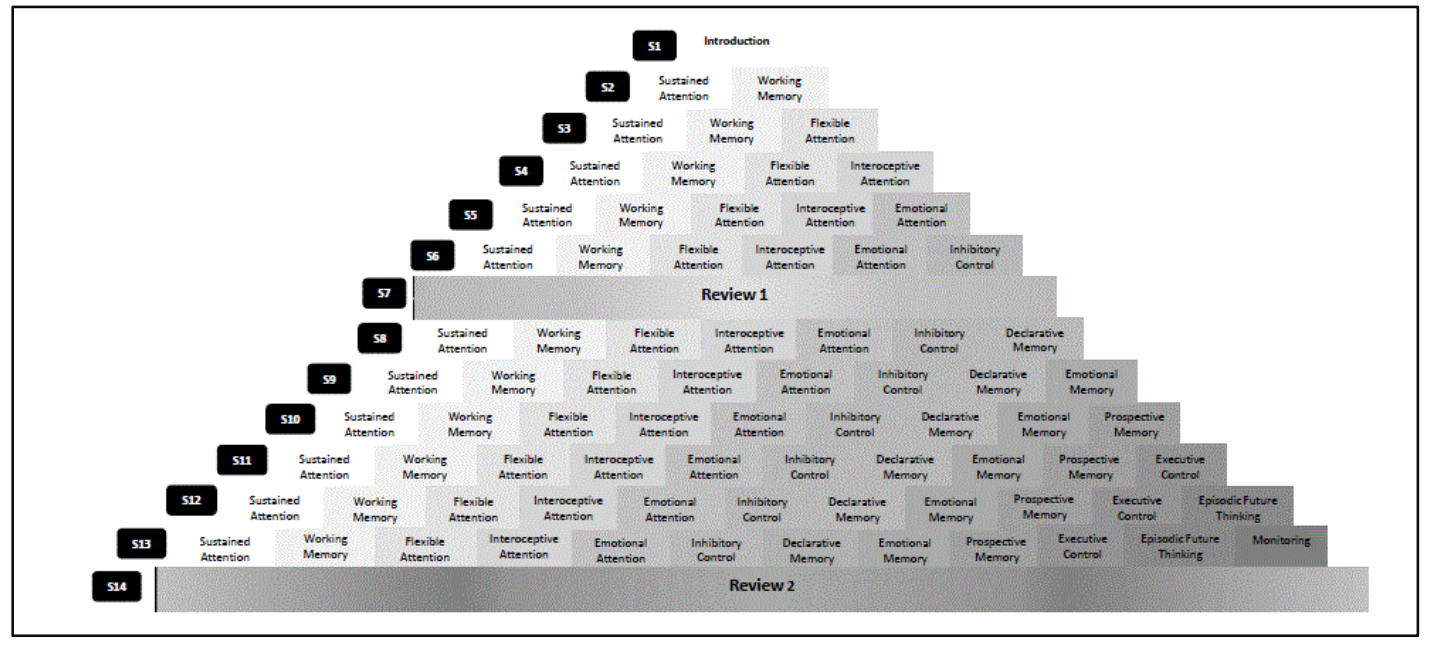

Figure 2.Architecture of the ProCoRe for 14 sessions. In each session a new cognitive domain is added to the previous one in trainings and exercises (accumulative architecture). The brightness of color indicates the level of complexity of the cognitive modules. The basic modules (e.g., sustained attention) marked with bright colors and the complex ones marked with dark colors (e.g., monitoring)

- Brain boxes: This section of the curriculum is a metacognitive training element which comes immediately after each brain exercise and informs participants about what cognitive functions were involved while they were doing the exercise/game (Matthews, Panganiban, Wells, Wohleber, \& Reinerman-Jones, 2019; Pozuelos et al., 2019). Moreover, in this section, participants can find information about similar situations to the exercise that may happen in the context of real-life. For example, the sample exercise mentioned above (Figure 3), is similar to what happens in real life when we are faced with a stressful event (e.g., scene of a car accident in which someone gets hurt seriously) and how we can manipulate this type of memory to lessen the emotional intensity of the related visual memory. 


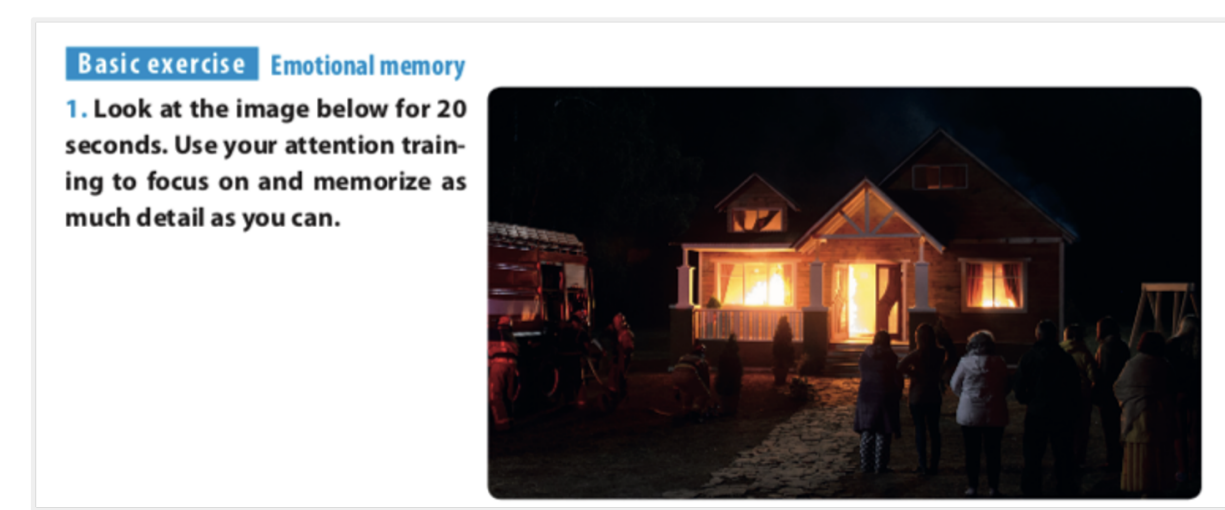

Figure 3. Sample of emotional memory exercise used in the brain exercise part (session 9). In this exercise, participants should try to expand their attention to memorize the peripheral details of the main event, which is a house fire (e.g., the number of house windows) and recall them later.

- Brain planner: This section is a time-management, self-monitoring or self-coaching part that trains participants how to monitor their emotions and behaviors as well as their progress toward their short-term and long-term goals, using a planner. This part of the curriculum is designed based on the work by (Plaete, De Bourdeaudhuij, Verloigne, \& Crombez, 2016). In each session, participants are expected to do some specific activities related to the cognitive functions that are covered in each specific session. For example, in the session related to flexible attention, participants should record the experiences/events when they do more than one task at the same time or switch between different tasks in their real-life. Participants should also write a short essay about their own experience of implementing the instructed compensatory strategies in their real life in the time between sessions.

In addition to the above-mentioned features, participants are assigned with some homework. Homework for each session is expected to provide an opportunity for participants to further practice what they have learned (Foster, Spence, \& Toglia, 2018). The Brain planner mentioned above is considered part of the homework that should be completed before each session.

\section{Cognitive Targets in ProCoRe Program}

ProCoRe has been developed to train five major cognitive functions that play critical role in building cognitive resilience (i.e., attention, interoception, saliency processing, memory, and executive control) through stimulating goal-directed behaviors (Kent et al.,2015). These major cognitive functions and their subdivisions are hierarchically presented through 14 sessions (figure 2). The details of each cognitive module are described as below:

- Attention: Various aspects of attention including focused and sustained attention, flexible attention, and emotional attention are targeted in ProCoRe. The "spotlight metaphor" is used to explain how the brain moves around and hunts the information in our environment and how this spotlight changes its size and direction in face of neutral and salient stimuli. During the training sessions, participants practice exercises that involve various aspects of attention and learn different brain tips including staying focused, mindfulness(Norris, Creem, Hendler, \& Kober, 2018), paraphrasing and self-talk(Hatzigeorgiadis \& Galanis, 2017). Therefore, the attention-related modules enable participants to stay focused and efficiently manage their attentional resources to capture important information and selectively filter irrelevant information.

- Interoception: Following the sessions that are mainly focused on exteroceptive attention (sessions 2-3), the next modules target interoception and interoceptive attention. During session 4, participants are trained to use the spotlight (the metaphor used for attention) to focus on their internal signals (e.g., heart rate, 
muscle tension, gut feeling and cold feeling in extremities) to consider these signals as a sign for specific emotion (e.g., anxious or anger) and use them to properly guide reciprocal behaviors(Khalsa et al., 2018). For example, they are trained to describe various physical and emotional changes that they may experience in face of an emotional event that may trigger impulsive decision and/or action (e.g., imagine that you see one of your close friends' pictures on Instagram hanging out at a party without telling you).

- Saliency processing: Several modules are devoted to explaining how saliency processing drives impulsive behaviors in face of incentive events and how this system becomes more prone to process stress-related stimuli more negatively (e.g., angry faces in crowd). The main goal pursued during these modules is to tune down the saliency attributed to emotional events by practicing strategies including gaze training(SanchezLopez, Everaert, Van Put, De Raedt, \& Koster, 2019), behavioral activation (Dimidjian et al., 2006), and different types of reappraisal techniques (Cutuli, 2014).

- Memory: Different dimensions of memory processing are targeted next. These include declarative memory, emotional memory and future (prospective) memory. In session 8, participants learn about episodic and semantic memories, their relationship to other cognitive domains introduced in previous sessions and their importance in learning and daily life. Session 9 is devoted to emotional memory, which concerns specifically salient stressful information such as previous experiences of being rejected by friends. In this session, participants learn how to deal with these emotional memories and reduce their emotional responses to these memories through learning about memory reconsolidation (Beckers \& Kindt, 2017). Session 10 is focused on memory for future goals and plans or prospective memory. The aim of this session is to train participants how to create and imagine a probable events that may happen in the future(Schacter, Benoit, \& Szpunar, 2017). With developing prospective memories on how to handle risky or stressful scenarios, participants become better equipped to face potential challenges. This is a preparation for the next sessions on self-control and decision making where participants should recruit a goal-oriented mental imagery to anticipate the short- and long-term consequences of one's decision. During the memory related sessions, various brain tips including mnemonic strategies, imagination, tunnel memory and episodic future thinking are discussed. The overall aim of the memory related sessions is to train participants for efficient use of memory for both past- and future-related memories, specifically those that may induce negative feelings once they are recalled or imagined.

- Executive control: In sessions 6, 10, 11 and 12, participants are trained in higher order cognitive functions including inhibition, future thinking, planning, self-monitoring, problem solving, and decision making and learn how to recruit and integrate them with basic cognitive functions to face risky, emotional, and stressful scenarios. To depict the interaction between these functions, two acronyms as "WFI" (session 11) and "SLIDE" (sessions 12-13) are being introduced. The WFI acronym includes Working memory, Flexibility and Inhibition and aims to depict how participants can use these functions to control their impulsive and negative habitual behaviors. For example, participants are asked to use WFI terms to explain how they can control their impulsive choices and resist offers to use drugs or alcohol. These sessions consist of various brain tips including development of "ready to go" plan and goal management training (Robertson, 1996). The second acronym is "SLIDE" in which participants learns how to Specify the problem, List possible solutions, Imagine outcomes, Do the chosen solution and Evolve the solution (update future plans based on previous outcomes). To apply the SLIDE acronym, participants need to be trained in episodic future thinking, mental imagery, brain storming, self-talk and verbalization strategies. All these strategies improve forethought, planning, and anticipation skills. The most prominent feature of the sessions targeting selfcontrol is using examples of real-life scenarios in which participants act that are closely related to what they may really do in the context of their real-life. For example, subjects are asked to imagine the scenario below and use SLIDE model to efficiently solve the problem.

"You have recently broken up with your very close friend. You have been feeling very down, lonely, and stressed since" 


\section{EASICoRe Model in ProCoRe for Resilience}

To depict how the five cognitive functions targeted in the ProCoRe, i.e., attention, saliency processing, memory, interoception, and executive control, interact with each other in face of impulsive/emotional/stressful triggers, we have developed a heuristic model termed as "EASICoRe" in ProCoRe (Figure 4). This neuroscience-informed model is used for both participants and trainers to frame their trainings through the ProCoRe program as an easily accessible heuristic.

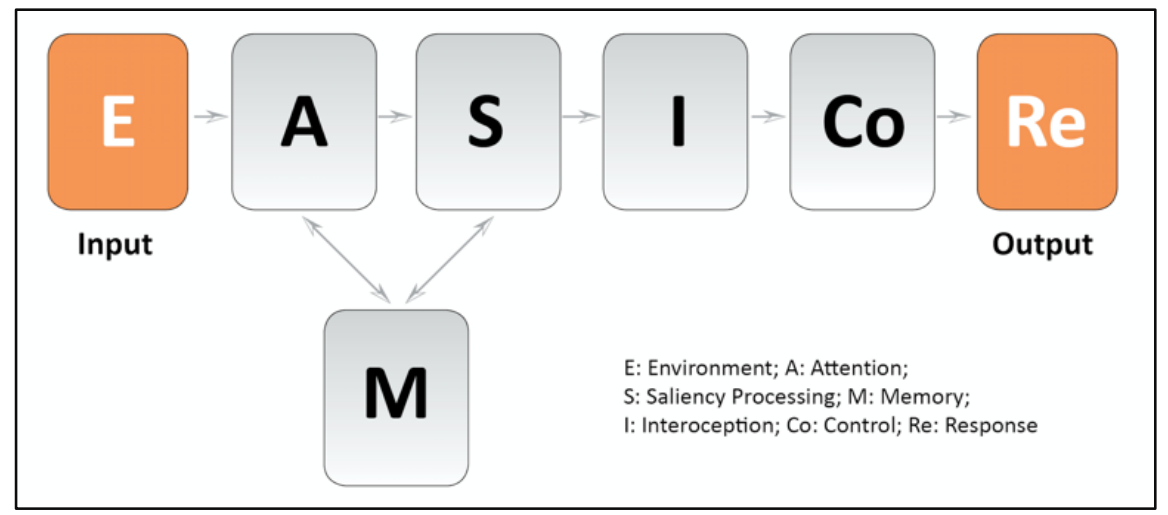

Figure 4. EASICoRe model as a neuroscience informed conceptual framework indicates the interaction between cognitive modules in ProCoRe which are activated in response to impulsive, emotional, and stressful triggers.

According to this model, once participants are exposed to an impulsive, emotional or stressful Environmental trigger, their Attentional resources are allocated to process different aspects of the trigger (e.g., emotional and physical), while their memory is biased towards relevant emotional memories. By integrating the information from attention and memory, the evaluation system starts processing the Saliency of incoming information and comparing them with subjective goals/values based on available appraisal schemas. At this moment, various somatic signals originate from within the body transferring information related to bodily experiences (e.g., heart rate, respiration rate, pupillary dilation, flushing, perspiration, nociceptive reflexes). These Interoceptive (somatic) signals contribute to emotional/appetitive experience and affect decision-making particularly under risk and uncertainty. Followed by evaluation, inhibitory Control may be activated to control impulsive desires and habits and direct behavior Response towards more goal-oriented action. Therefore, EASICoRe can be considered as a neuroscience-informed framework which defines cognitive targets in the dynamic response to stressful, emotional, and appetitive cues. It is assumed that the proper functioning of each cognitive step as well as their interactions, increases selfregulation and as a consequence improves resilience.

Due to the hierarchical structure of ProCoRe, in which cognitive modules are organized from the basic to the complex level, participants are presented with the EASICoRe model sequentially through the sessions and practice each module gradually in terms of real-life context. For example, by training with the attentionskills module, they learn how to use relevant brain tips to cope with attentional bias, or after learning the memory-related module, they start using relevant strategies to reduce negative feelings when they recall stressful events. Figure 5 indicates how EASICoRe related skills interact with each other in face of emotional and impulsive stimuli to protect the individual who is facing stressful conditions. 


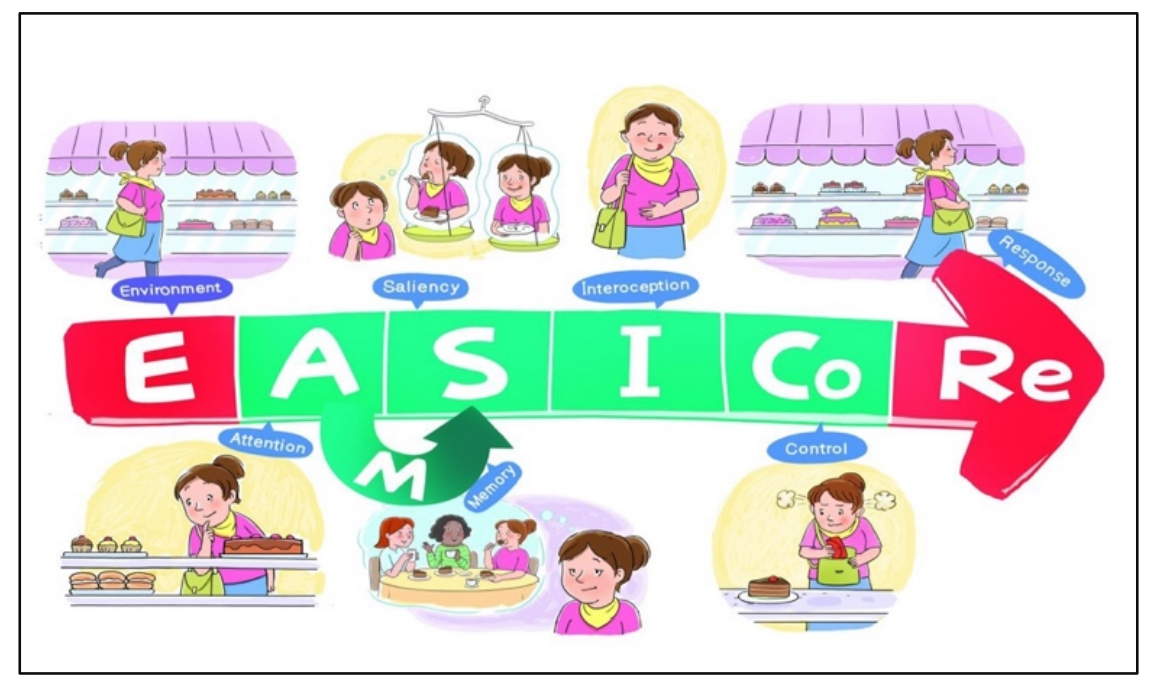

Figure 5. The interaction of EASICoRe related skills in face of emotional and impulsive stimuli. Participants are introduced with the basics of the EASICoRe model in the first session and gradually over the next sessions, they learn how to use this simple heuristic to use the ProCoRe trainings in real life scenarios.

\section{Public Health and Clinical Implication of ProCoRe for Drug Use Prevention}

Enhancing resilience in order to increase adaptive behaviors in face of stressful and emotional events, can be extended to risky situations that may involve illicit drugs or alcohol use. To enhance the coverage for these situations, in an experiment, we added additional modules to ProCoRe to provide relevant pictorial and verbal scenarios. In the additional modules, participants learn through the EASICoRe model how substance use disorders develop with repeated drug use, which hijacks neural circuits normally involved in healthy motivational behaviors like eating to motivate continued drug use and development of addiction. Additional cartoons and metaphors illustrate this process, such as the pictorial metaphor of "brain circuit thief" used to indicate how the brain is hijacked and controlled by substance use (Figure 6). Moreover, to increase participants' knowledge about the effects of chronic substance use on the brain, additional modules provide neuroscience-informed educational materials that depict alterations in brain structure and functions. The synergistic interaction of these additional substance use prevention modules to the ProCoRe and their effectiveness as a prevention intervention for substance use disorders needs to be experimentally quantified by future studies.

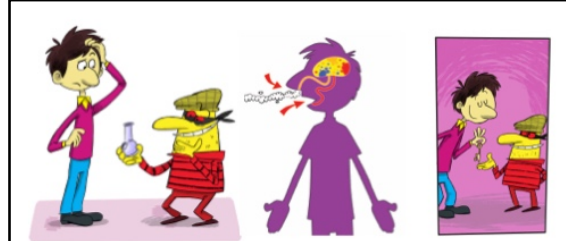

(a)

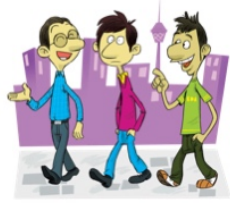

(e) (b)

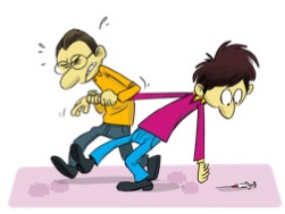

(f)

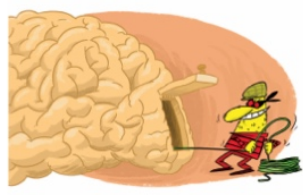

(c)

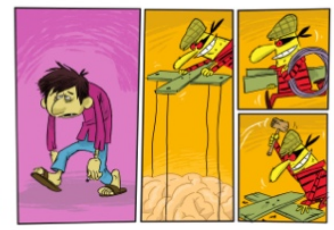

(g)

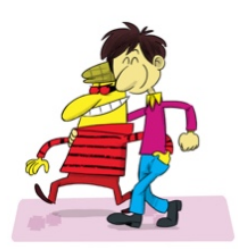

(d)

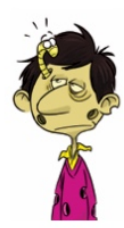

(h)

Figure 6. Sample of a cartoon used in the neuroscience-informed education part related to drug addiction. 


\section{Feasibility Study with ProCoRe}

To examine the fit of ProCoRe in the real world, HE and TR with a group of collaborators are running two feasibility studies in two separate high schools as an education curriculum on Iranian $11^{\text {th }}$ grader during an academic year (2019-2020). ProCoRe consists of 14, 90-min group sessions which are manualized and mentored by trained coaches. The outcome measures are change of resilience and emotion regulation indexed by self-reports and neuropsychological assessments of executive functions (e.g., inhibition, working memory and flexibility) from pre- to post intervention. Academic achievement measured by student's exam scores over the year and acceptability of the program are also assessed.

\section{Challenges and Future Directions}

From a neurocognitive perspective, cognitive resilience is embedded in a set of brain structures and cognitive functions which collectively contribute to the regulation of emotions, inhibition of impulses, motivation and adaptive behaviors that result in positive outcomes (Figure 7). In light of neuroplasticity and the trainable nature of cognitive functions(Ballesteros, Voelcker-Rehage, \& Bherer, 2018; Green \& Bavelier, 2008). ProCoRe has been developed as a modular training program inspired by a neuroscienceinformed framework, EASICoRe. We hypothesize that this program improves cognitive resilience through targeting different cognitive functions involved in emotion-regulation, impulse-control, learning, stressmanagement, flexibility and problem-solving. Following the developmental phase of the program, the authors are testing the real-world efficacy of this training program with adolescents by focusing on measurable neurocognitive and behavioral markers. It would be critical use a comprehensive neuropsychological battery, to test the efficacy of this program with diverse populations of youth at risk of substance misuse and other maladaptive behaviors, including different racial and ethnic groups, those who were raised in poverty, individuals from single parent families, as well as youth who identify as lesbian, gay, bisexual and transgender (LGBT).Our next step is to prepare the computerized and cell-phone version of the ProCoRe to make it more accessible and interactive for individual application. Translation of neuroscience evidence into practical applications, makes ProCoRe trainings more real-life friendly and provides more opportunities for metacognitive awareness to reshape life skills. The ultimate goal is to scale the intervention to enhance population level resilience of adolescents and young adults so they can avoid high risk behaviors at the time of stress. We welcome any collaborations to develop ProCoRe materials in different languages and assess the efficacy of the the culturally adapted program in different communities.

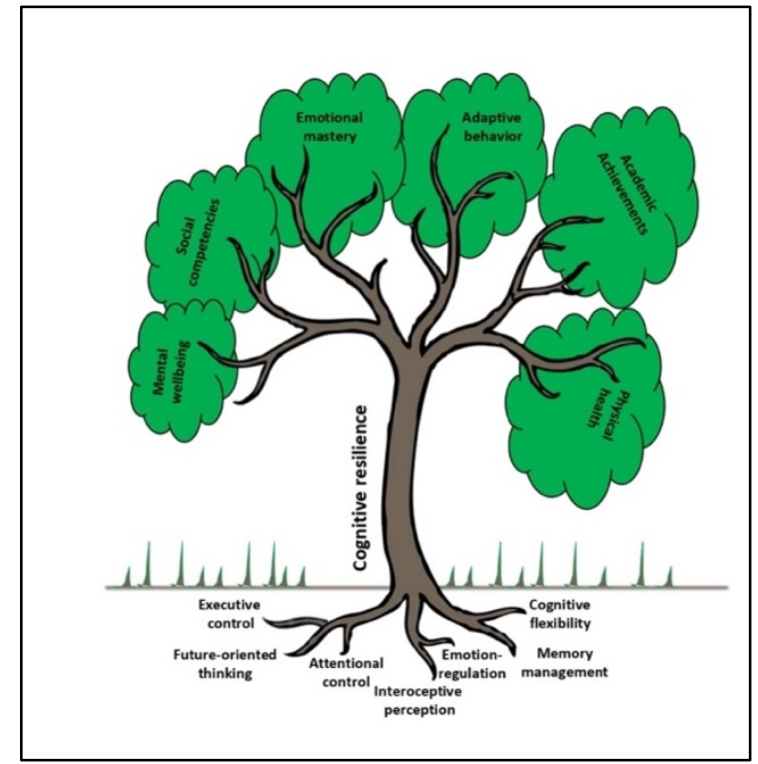

Figure 7. Visualizing cognitive resilience with the concept of green tree (roots are metaphor for cognitive functions and branches are metaphor for outcomes) 


\section{Competing interests}

Hamed Ekhtiari, Tara Rezapour, Martin P. Paulus and Robin L. Aupperle were involved with the ProCoRe program development.

\section{Acknowledgment}

The authors would like to thank Seyed Naeim Tadayon Nabavi Fadafan (illustrator), Laleh Ziai (illustrator) and Mohsen Farhadi (graphic designer) for their contribution in creating cartoons and graphic design of ProCoRe program.

\section{References}

Ambelu, A., Mulu, T., Seyoum, A., Ayalew, L., \& Hildrew, S. (2019). Resilience dynamics after interventions made among school children of rural Ethiopia. Heliyon, 5(4), e01464. doi:10.1016/j.heliyon.2019.e01464

Artuch-Garde, R., Gonzalez-Torres, M. D. C., de la Fuente, J., Vera, M. M., Fernandez-Cabezas, M., \& Lopez-Garcia, M. (2017). Relationship between Resilience and Self-regulation: A Study of Spanish Youth at Risk of Social Exclusion. Front Psychol, 8, 612. doi:10.3389/fpsyg.2017.00612

Ballesteros, S., Voelcker-Rehage, C., \& Bherer, L. (2018). Editorial: Cognitive and Brain Plasticity Induced by Physical Exercise, Cognitive Training, Video Games, and Combined Interventions. Front Hum Neurosci, 12, 169. doi:10.3389/fnhum.2018.00169

Beckers, T., \& Kindt, M. (2017). Memory Reconsolidation Interference as an Emerging Treatment for Emotional Disorders: Strengths, Limitations, Challenges, and Opportunities. Annu Rev Clin Psychol, 13, 99-121. doi:10.1146/annurev-clinpsy-032816-045209

Bjork, J. M., Smith, A. R., Danube, C. L., \& Hommer, D. W. (2007). Developmental differences in posterior mesofrontal cortex recruitment by risky rewards. $J$ Neurosci, 27(18), 4839-4849. doi:10.1523/JNEUROSCI.5469-06.2007

Blair, C., \& Raver, C. C. (2015). School readiness and self-regulation: a developmental psychobiological approach. Annu Rev Psychol, 66, 711-731. doi:10.1146/annurev-psych-010814-015221

Blakemore, S. J., \& Robbins, T. W. (2012). Decision-making in the adolescent brain. Nat Neurosci, 15(9), 1184-1191. doi:10.1038/nn.3177

Cambron, C., Kosterman, R., Catalano, R. F., Guttmannova, K., Herrenkohl, T. I., Hill, K. G., \& Hawkins, J. D. (2017). The Role of Self-Regulation in Academic and Behavioral Paths to a High School Diploma. J Dev Life Course Criminol, 3(3), 304-325. doi:10.1007/s40865-017-0066-5

Casey, B., Jones, RM., Hare, TA. (2008). The Adolescent Brain. Ann N Y Acad Sci, 1124, 111-126.

Casey, B. J., Galvan, A., \& Hare, T. A. (2005). Changes in cerebral functional organization during cognitive development. Curr Opin Neurobiol, 15(2), 239-244. doi:10.1016/j.conb.2005.03.012

Chadda, R. K. (2018). Youth \& mental health: Challenges ahead. Indian J Med Res, 148(4), 359-361. doi:10.4103/ijmr.IJMR_1585_18

Clark, D. B., \& Winters, K. C. (2002). Measuring risks and outcomes in substance use disorders prevention research. Journal of Consulting and Clinical Psychology, 70(6), 1207-1223. doi:10.1037/0022006x.70.6.1207

Clarke, A. M., Bunting, B., \& Barry, M. M. (2014). Evaluating the implementation of a school-based emotional well-being programme: a cluster randomized controlled trial of Zippy's Friends for children in disadvantaged primary schools. Health Educ Res, 29(5), 786-798. doi:10.1093/her/cyu047

Claus, E. D., Feldstein Ewing, S. W., Magnan, R. E., Montanaro, E., Hutchison, K. E., \& Bryan, A. D. (2018). Neural mechanisms of risky decision making in adolescents reporting frequent alcohol and/or marijuana use. Brain Imaging Behav, 12(2), 564-576. doi:10.1007/s11682-017-9723-x 
Cutuli, D. (2014). Cognitive reappraisal and expressive suppression strategies role in the emotion regulation: an overview on their modulatory effects and neural correlates. Front Syst Neurosci, 8, 175. doi:10.3389/fnsys.2014.00175

Dantzer, R., Cohen, S., Russo, S. J., \& Dinan, T. G. (2018). Resilience and immunity. Brain Behav Immun, 74, 28-42. doi:10.1016/j.bbi.2018.08.010

Debenham, J., Birrell, L., Champion, K., Askovic, M., \& Newton, N. (2020). A pilot study of a neuroscience-based, harm minimisation programme in schools and youth centres in Australia. BMJ Open, 10(2), e033337. doi:10.1136/bmjopen-2019-033337

Dick, D. M., Adkins, A. E., \& Kuo, S. I. (2016). Genetic influences on adolescent behavior. Neurosci Biobehav Rev, 70, 198-205. doi:10.1016/j.neubiorev.2016.07.007

Dimidjian, S., Hollon, S. D., Dobson, K. S., Schmaling, K. B., Kohlenberg, R. J., Addis, M. E., . . . Jacobson, N. S. (2006). Randomized trial of behavioral activation, cognitive therapy, and antidepressant medication in the acute treatment of adults with major depression. $J$ Consult Clin Psychol, 74(4), 658-670. doi:10.1037/0022-006X.74.4.658

Donovan, J. E. (2004). Adolescent alcohol initiation: A review of psychosocial risk factors. Journal of adolescent health, 35(6), 529. e527-529. e518.

Dow, S. J., \& Kelly, J. F. (2013). Listening to youth: Adolescents' reasons for substance use as a unique predictor of treatment response and outcome. Psychology of Addictive Behaviors, 27(4), 1122.

Dray, J., Bowman, J., Freund, M., Campbell, E., Wolfenden, L., Hodder, RK., Wiggers, J. (2014). Improving adolescent mental health and resilience through a resilience-based intervention in schools: study protocol for a randomised controlled trial. Trials, 15(289), 1-9.

Dyrbye, L. N., West, C. P., Richards, M. L., Ross, H. J., Satele, D., \& Shanafelt, T. D. (2016). A randomized, controlled study of an online intervention to promote job satisfaction and well-being among physicians. Burnout Research, 3(3), 69-75. doi:10.1016/j.burn.2016.06.002

Ekhtiari, H., Rezapour, T., Aupperle, R. L., \& Paulus, M. P. (2017). Neuroscience-informed psychoeducation for addiction medicine: A neurocognitive perspective. Prog Brain Res, 235, 239264. doi:10.1016/bs.pbr.2017.08.013

Foster, E. R., Spence, D., \& Toglia, J. (2018). Feasibility of a cognitive strategy training intervention for people with Parkinson's disease. Disabil Rehabil, 40(10), 1127-1134. doi:10.1080/09638288.2017.1288275

Franklin, T. B., Saab, B. J., \& Mansuy, I. M. (2012). Neural mechanisms of stress resilience and vulnerability. Neuron, 75(5), 747-761. doi:10.1016/j.neuron.2012.08.016

Galvan, A., Hare, T., Voss, H., Glover, G., \& Casey, B. J. (2007). Risk-taking and the adolescent brain: who is at risk? Dev Sci, 10(2), F8-F14. doi:10.1111/j.1467-7687.2006.00579.x

Galvan, A., Hare, T. A., Parra, C. E., Penn, J., Voss, H., Glover, G., \& Casey, B. J. (2006). Earlier development of the accumbens relative to orbitofrontal cortex might underlie risk-taking behavior in adolescents. $J$ Neurosci, 26(25), 6885-6892. doi:10.1523/JNEUROSCI.1062-06.2006

Giedd, J., Blumenthal, J., Jeffries, NO., Castellanos, FX., Liu, H., Zijdenbos, A., Paus, T., Evans, AC., Rapoport, JL. (1999). Brain development during childhood and adolescence: a longitudinal MRI study. Nat Neurosci, 2(10), 861-863.

Goodman, M. J., \& Schorling, J. B. (2012). A mindfulness course decreases burnout and improves wellbeing among healthcare providers. Int J Psychiatry Med, 43(2), 119-128. doi:10.2190/PM.43.2.b

Green, C. S., \& Bavelier, D. (2008). Exercising your brain: a review of human brain plasticity and traininginduced learning. Psychol Aging, 23(4), 692-701. doi:10.1037/a0014345

Grossman, D., Neckerman, HJ., Koepsell, TD., Liu, PY., Asher, KN., Beland, K., Frey, K., Rivara, FP. (1997). Effectiveness of a violence prevention curriculum among children in elementary school. A randomized controlled trial. JAMA, 277(20), 1605-1611.

Hartley, C. A., \& Somerville, L. H. (2015). The neuroscience of adolescent decision-making. Curr Opin Behav Sci, 5, 108-115. doi:10.1016/j.cobeha.2015.09.004

Hatzigeorgiadis, A., \& Galanis, E. (2017). Self-talk effectiveness and attention. Curr Opin Psychol, 16, 138-142. doi:10.1016/j.copsyc.2017.05.014 
Iadipaolo, A. S., Marusak, H. A., Paulisin, S. M., Sala-Hamrick, K., Crespo, L. M., Elrahal, F., .. . Rabinak, C. A. (2018). Distinct neural correlates of trait resilience within core neurocognitive networks in at-risk children and adolescents. Neuroimage Clin, 20, 24-34. doi:10.1016/j.nicl.2018.06.026

Jaworska, N., \& MacQueen, G. (2015). Adolescence as a unique developmental period. J Psychiatry Neurosci, 40(5), 291-293. doi:10.1503/jpn.150268

Joyce, S., Shand, F., Tighe, J., Laurent, S. J., Bryant, R. A., \& Harvey, S. B. (2018). Road to resilience: a systematic review and meta-analysis of resilience training programmes and interventions. $B M J$ Open, 8(6), e017858. doi:10.1136/bmjopen-2017-017858

Jurewicz, I. (2015). Mental health in young adults and adolescents - supporting general physicians to provide holistic care. Clin Med (Lond). 15(2), 151-154.

Kahn, J. R., Collinge, W., \& Soltysik, R. (2016). Post-9/11 Veterans and Their Partners Improve Mental Health Outcomes with a Self-directed Mobile and Web-based Wellness Training Program: A Randomized Controlled Trial. J Med Internet Res, 18(9), e255. doi:10.2196/jmir.5800

Kent, M., Rivers, C. T., \& Wrenn, G. (2015). Goal-Directed Resilience in Training (GRIT): A Biopsychosocial Model of Self-Regulation, Executive Functions, and Personal Growth (Eudaimonia) in Evocative Contexts of PTSD, Obesity, and Chronic Pain. Behav Sci (Basel), 5(2), 264-304. doi:10.3390/bs5020264

Khalsa, S. S., Adolphs, R., Cameron, O. G., Critchley, H. D., Davenport, P. W., Feinstein, J. S., . . . Interoception Summit, p. (2018). Interoception and Mental Health: A Roadmap. Biol Psychiatry Cogn Neurosci Neuroimaging, 3(6), 501-513. doi:10.1016/j.bpsc.2017.12.004

Knudsen, E. (2004). Sensitive periods in the development of the brain and behavior. J Cogn Neurosci., $16(8), 1412-1425$.

Labriole, M. (2010). Promoting Brain-Science Literacy in the K-12 Classroom By Michaela Labriole. Cerebrum: the Dana forum on brain science, 15, 1-14.

Lee, F. S., Heimer, H., Giedd, J. N., Lein, E. S., Sestan, N., Weinberger, D. R., \& Casey, B. J. (2014). Mental health. Adolescent mental health--opportunity and obligation. Science, 346(6209), 547-549. doi:10.1126/science. 1260497

Liu, H., Zhang, C., Ji, Y., \& Yang, L. (2018). Biological and Psychological Perspectives of Resilience: Is It Possible to Improve Stress Resistance? Front Hum Neurosci, 12, 326. doi:10.3389/fnhum.2018.00326

Loprinzi CE, P. K., Schroeder DR, Sood A. (2011). Stress Management and Resilience Training (SMART) program to decrease stress and enhance resilience among breast cancer survivors: a pilot randomized clinical trial. Clin Breast Cancer., 11(6), 364-368.

Luthar SS, C. D., Becker B. . (2000). The Construct of Resilience: A Critical Evaluation and Guidelines

for Future Work. Child Dev, 71(3), 543-562.

Mache, S., Bernburg, M., Baresi, L., \& Groneberg, D. A. (2016). Evaluation of self-care skills training and solution-focused counselling for health professionals in psychiatric medicine: a pilot study. Int $J$ Psychiatry Clin Pract, 20(4), 239-244. doi:10.1080/13651501.2016.1207085

Masten, A. S., \& Barnes, A. J. (2018). Resilience in Children: Developmental Perspectives. Children (Basel), 5(7). doi:10.3390/children5070098

Matthews, G., Panganiban, A. R., Wells, A., Wohleber, R. W., \& Reinerman-Jones, L. E. (2019). Metacognition, Hardiness, and Grit as Resilience Factors in Unmanned Aerial Systems (UAS) Operations: A Simulation Study. Front Psychol, 10, 640. doi:10.3389/fpsyg.2019.00640

McClowry, S. G., Snow, D. L., Tamis-Lemonda, C. S., \& Rodriguez, E. T. (2010). Testing the Efficacy of INSIGHTS on Student Disruptive Behavior, Classroom Management, and Student Competence in Inner City Primary Grades. School Ment Health, 2(1), 23-35. doi:10.1007/s12310-009-9023-8

McCraty R, A. M. (2012). Resilience Training Program Reduces Physiological and Psychological Stress in Police Officers. Glob Adv Health Med, 1(5), 44-66.

McGue M, I. W., Krueger R. (2006). The association of early adolescent problem behavior and adult psychopathology: a multivariate behavioral genetic perspective. Behav Genet, 36(4), 591-602. 
Mealer, M., Conrad, D., Evans, J., Jooste, K., Solyntjes, J., Rothbaum, B., \& Moss, M. (2014). Feasibility and acceptability of a resilience training program for intensive care unit nurses. Am J Crit Care, 23(6), e97-105. doi:10.4037/ajcc2014747

Nigg, J. T. (2017). Annual Research Review: On the relations among self-regulation, self-control, executive functioning, effortful control, cognitive control, impulsivity, risk-taking, and inhibition for developmental psychopathology. $J$ Child Psychol Psychiatry, 58(4), 361-383. doi:10.1111/jepp. 12675

Nishida, A., Richards, M., \& Stafford, M. (2016). Prospective associations between adolescent mental health problems and positive mental wellbeing in early old age. Child Adolesc Psychiatry Ment Health, 10, 12. doi:10.1186/s13034-016-0099-2

Norris, C. J., Creem, D., Hendler, R., \& Kober, H. (2018). Brief Mindfulness Meditation Improves Attention in Novices: Evidence From ERPs and Moderation by Neuroticism. Front Hum Neurosci, 12, 315. doi:10.3389/fnhum.2018.00315

Pandey A, H. D., Das S, Goddings AL, Blakemore SJ, Viner RM. (2018). Effectiveness of Universal Selfregulation-Based Interventions in Children and Adolescents: A Systematic Review and Metaanalysis. JAMA Pediatr., 172(6), 566-575.

Plaete, J., De Bourdeaudhuij, I., Verloigne, M., \& Crombez, G. (2016). The use and evaluation of selfregulation techniques can predict health goal attainment in adults: an explorative study. PeerJ, 4, e1666. doi:10.7717/peerj.1666

Pozuelos, J. P., Combita, L. M., Abundis, A., Paz-Alonso, P. M., Conejero, A., Guerra, S., \& Rueda, M. R. (2019). Metacognitive scaffolding boosts cognitive and neural benefits following executive attention training in children. Dev Sci, 22(2), e12756. doi:10.1111/desc.12756

Ram, D., Chandran, S., Sadar, A., \& Gowdappa, B. (2019). Correlation of Cognitive Resilience, Cognitive Flexibility and Impulsivity in Attempted Suicide. Indian J Psychol Med, 41(4), 362-367. doi:10.4103/IJPSYM.IJPSYM_189_18

Richter, A., Kramer, B., Diekhof, E. K. ., \& Gruber, O. (2019). Resilience to adversity is associated with increased activity and connectivity in the VTA and hippocampus. Neuroimage Clin, 23, 101920. doi:10.1016/j.nicl.2019.101920

Riggs, N., Greenberg, MT., Kusché, CA., Pentz, MA. (2006). The mediational role of neurocognition in the behavioral outcomes of a social-emotional prevention program in elementary school students: effects of the PATHS Curriculum. Prev Sci., 7(1), 91-102.

Rossignoli-Palomeque, T., Perez-Hernandez, E., \& Gonzalez-Marques, J. (2018). Brain Training in Children and Adolescents: Is It Scientifically Valid? Front Psychol, 9, 565. doi:10.3389/fpsyg.2018.00565

Rossignoli-Palomeque, T., Quiros-Godoy, M., Perez-Hernandez, E., \& Gonzalez-Marques, J. (2019). Schoolchildren's Compensatory Strategies and Skills in Relation to Attention and Executive Function App Training. Front Psychol, 10, 2332. doi:10.3389/fpsyg.2019.02332

Roughan, L., \& Hadwin, J. A. (2011). The impact of working memory training in young people with social, emotional and behavioural difficulties. Learning and Individual Differences, 21(6), 759-764. doi:10.1016/j.lindif.2011.07.011

Russo, S. J., Murrough, J. W., Han, M. H., Charney, D. S., \& Nestler, E. J. (2012). Neurobiology of resilience. Nat Neurosci, 15(11), 1475-1484. doi:10.1038/nn.3234

Sanchez-Lopez, A., Everaert, J., Van Put, J., De Raedt, R., \& Koster, E. H. W. (2019). Eye-gaze contingent attention training (ECAT): Examining the causal role of attention regulation in reappraisal and rumination. Biol Psychol, 142, 116-125. doi:10.1016/j.biopsycho.2019.01.017

Schacter, D. L., Benoit, R. G., \& Szpunar, K. K. (2017). Episodic Future Thinking: Mechanisms and Functions. Curr Opin Behav Sci, 17, 41-50. doi:10.1016/j.cobeha.2017.06.002

Shure, M. B. (1993). I can problem solve (ICPS): Interpersonal cognitive problem solving for young children. Early Child Development and Care, 96(1), 49-64. doi:10.1080/0300443930960106 
Sowell, E. R., Peterson, B. S., Thompson, P. M., Welcome, S. E., Henkenius, A. L., \& Toga, A. W. (2003). Mapping cortical change across the human life span. Nat Neurosci, 6(3), 309-315. doi:10.1038/nn1008

Spear, L. (2000). The adolescent brain and age-related behavioral manifestations. Neurosci Biobehav Rev, 24(4), 417-463.

Stanger, C., Budney, A. J., \& Bickel, W. K. (2013). A developmental perspective on neuroeconomic mechanisms of contingency management. Psychol Addict Behav, 27(2), 403-415. doi: $10.1037 / \mathrm{a} 0028748$

Steinberg, L. (2004). Risk taking in adolescence: what changes, and why? Ann N Y Acad Sci., 1021, 51-58.

Steinberg, L., Graham, S., O'Brien, L., Woolard, J., Cauffman, E., Banich, M. (2009). Age differences in future orientation and delay discounting. Child Dev 80(1), 28-44.

Titus, J. C., Godley, S. H., \& White, M. K. (2007). A post-treatment examination of adolescents' reasons for starting, quitting, and continuing the use of drugs and alcohol. Journal of child \& adolescent substance abuse, 16(2), 31-49.

Victoria Cerezo, M., Ortiz-Tallo, M., Cardenal, V., \& De La Torre-Luque, A. (2014). Positive psychology group intervention for breast cancer patients: a randomised trial. Psychol Rep, 115(1), 44-64. doi:10.2466/15.20.PR0.115c17z7

Vujovic, L., \& Urry, H. L. (2018). Emotion Regulation Compensation Following Situation Selection Failure. Sci Rep, 8(1), 5411. doi:10.1038/s41598-018-23654-2

Webster-Stratton, C., Jamila Reid, M., \& Stoolmiller, M. (2008). Preventing conduct problems and improving school readiness: evaluation of the Incredible Years Teacher and Child Training Programs in high-risk schools. J Child Psychol Psychiatry, 49(5), 471-488. doi:10.1111/j.14697610.2007.01861.x

Wetherill, R., \& Tapert, S. F. (2013). Adolescent brain development, substance use, and psychotherapeutic change. Psychol Addict Behav, 27(2), 393-402. doi:10.1037/a0029111

Wu, G., Feder, A., Cohen, H., Kim, J. J., Calderon, S., Charney, D. S., \& Mathe, A. A. (2013). Understanding resilience. Front Behav Neurosci, 7, 10. doi:10.3389/fnbeh.2013.00010

Yamamoto, T., Matsumoto, Y., \& Bernard, M. E. (2017). Effects of the cognitive-behavioral You Can Do It! Education program on the resilience of Japanese elementary school students: A preliminary investigation. International Journal of Educational Research, 86, 50-58. doi:10.1016/j.ijer.2017.08.006 